\title{
Merlin, the liver wizard
}

The two most common types of liver cancer are hepatocellular carcinoma (HCC) and cholangiocarcinoma (CC). Mouse models of liver cancer, based on either chemical induction or genetic engineering, usually reproduce HCC but rarely reproduce CC. One exception is mice that are heterozygous for the tumour suppressor neurofibromatosis type 2 (Nf2), which develop both types of tumours.

Andrea McClatchey and collaborators were interested in studying how the Nf2-encoded protein Merlin controls proliferation in the developing as well as the adult mouse liver and how its absence promotes tumorigenesis. To that end, they developed two types of conditional $N f 2$-knockout mouse in which Nf2 could be deleted in the liver at different developmental stages. Deletion of $N f 2$ during liver development led to a rapid enlargement of the liver, which

These results propose a potential role for Merlin in regulating - in a hippo-independent manner - the proliferation of liver progenitor cells from which $\mathrm{HCC}$ and CC could arise. was attributed to expansion of oval cells (OCs) - also known as liver progenitor cells - but not hepatocytes, indicating that only progenitor cells are sensitive to the proliferation triggered by $N f 2$ loss.

What mechanisms might contribute to this phenotype? The authors had previously shown that Merlin regulates epidermal growth factor receptor (EGFR) internalization, so they investigated the effects of Merlin loss on OC proliferation that is regulated by the EGFR signalling pathway. Treatment of

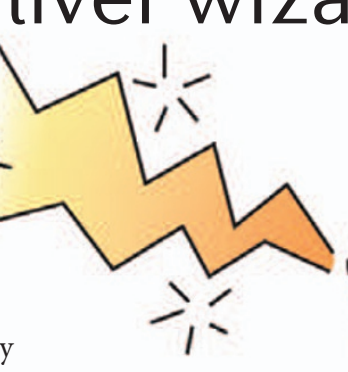

mice harbouring Nf2-deficient livers with the EGFR inhibitor erlonitib resulted in a dramatic reduction in liver size and cell proliferation, indicating that EGFR signalling is necessary for the expansion of $\mathrm{Nf}^{-/-}$progenitor cells.

This expansion of OCs invariably led to the development of HCC, CC and metastases in the lung, similar to $\mathrm{Nf2} 2^{+/-}$mice. However, when $N f 2$ was deleted in the adult mouse liver, tumours appeared only after stimulation by partial hepactectomy. This suggests that hepatectomy triggers the proliferation of $\mathrm{Nf2^{-/- }}$ progenitor cells that would normally be quiescent in the adult liver. It also suggests that the role of Merlin in the liver is restricted to the control of proliferation of progenitor cells. The authors showed that the injection of $\mathrm{Nf}^{-/-}$ hepatoblasts into immunocompromised mice led to OC hyperplasia and the development of tumours with CC features.

Interestingly, although Merlin has been reported to regulate the Hippo signalling pathway and this pathway has recently been implicated in liver size control and cancer, the authors failed to find evidence that Merlin alone regulates the Hippo pathway in liver progenitor cells, suggesting that Merlin and Hippo might regulate liver size through different cell types.

These results propose a potential role for Merlin in regulating - in a hippo-independent manner - the proliferation of liver progenitor cells from which HCC and CC could arise.

Teresa Villanueva

ORIGINAL RESEARCH PAPER Benhamouche, S. et al. Nf2/Merlin controls progenitor homeostasis and tumorigenesis in the liver. Genes Dev. 24, 1718-1730 (2010) 